\title{
Noninvasive functional photoacoustic tomography of blood-oxygen saturation in the brain
}

Xueding Wang, Geng Ku, Xueyi Xie, Yiwen Wang, George Stoica, et al.

Xueding Wang, Geng Ku, Xueyi Xie, Yiwen Wang, George Stoica, Lihong V. Wang, "Noninvasive functional photoacoustic tomography of blood-oxygen saturation in the brain," Proc. SPIE 5320, Photons Plus Ultrasound: Imaging and Sensing, (12 July 2004); doi: 10.1117/12.532440

SPIE. Event: Biomedical Optics 2004, 2004, San Jose, CA, United States 


\title{
Non-invasive functional photoacoustic tomography of blood oxygen saturation in the brain
}

\author{
Xueding Wang ${ }^{\mathrm{a}}$, Geng Ku ${ }^{\mathrm{a}}$, Xueyi Xie ${ }^{\mathrm{a}}$, Yiwen Wang ${ }^{\mathrm{a}}$, George Stoica ${ }^{\mathrm{b}}$, \\ and Lihong V. Wang ${ }^{* a}$ \\ ${ }^{a}$ Optical Imaging Laboratory, Department of Biomedical Engineering, Texas A\&M University, \\ 3120 TAMU, College Station, Texas 77843-3120 \\ ${ }^{b}$ Department of Pathobiology, Texas A\&M University, College Station, TX 77843-5547
}

\begin{abstract}
Since optical contrast is sensitive to functional parameters, including the hemoglobin oxygen saturation and the total concentration of hemoglobin, imaging based on optical contrast has been widely employed for the real-time monitoring of tissue oxygen consumption and hemodynamics in biological tissues. However, due to the overwhelming scattering of light in tissues, traditional optical imaging modalities cannot provide satisfactory spatial resolution. Functional photoacoustic tomography is a novel technique that combines high optical contrast and high ultrasonic resolution. Here, we present our study of a laser-based photoacoustic technique that, for the first time to our knowledge, monitors blood oxygenation in the rat brain in vivo. The cerebral blood oxygenation in the rat brain was imaged by photoacoustic detection at two wavelengths. The change in the hemoglobin oxygen saturation in the brain vessels as a result of the alternation from hyperoxia status to hypoxia status was visualized successfully with satisfactory spatial resolution. This work demonstrates that photoacoustic technique, based on the spectroscopic absorption of oxy- and deoxy-hemoglobin, can provide accurate functional imaging of cerebral blood oxygenation in the small-animal brain non-invasively with the skin and skull intact.
\end{abstract}

Keywords: photoacoustic, optoacoustic, spectroscopic absorption, hemoglobin oxygen saturation, cerebral blood oxygenation, functional neuroimaging

\section{INTRODUCTION}

The study of functional changes in regional blood oxygenation (hemoglobin oxygen saturation) and blood flow (total concentration of hemoglobin) is important to diagnostic imaging and therapeutic monitoring. Despite years of effort, however, no imaging modality has been developed previously that is clinically feasible for the accurate, continuous and non-invasive monitoring of hemoglobin oxygen saturation in distant organs, such as brain. Since the brain is highly responsive to changes in blood oxygenation, rapid high-resolution mapping of oxygenation is of great significance in neurophysiology, neuropathology, and neurotherapy, for example in visualizing the functional parameters of brain cancers and traumatic brain injuries, monitoring ischemia and shock, and studying neural activities. Near-infrared spectroscopy (NIRS), based on the spectroscopic difference between oxy- and deoxy-hemoglobin, has long been explored for the non-invasive measurement of blood oxygenation. ${ }^{1-3}$ However, because of the overwhelming scattering of light in biological tissues, this technique has so far proved incapable of providing satisfactory resolution and accuracy for imaging deep tissues beyond the skin.

Photoacoustic tomography (PAT), also named as optoacoustic tomography or thermoacoustic tomography), ${ }^{4-9}$ is a novel non-invasive imaging modality that combines the high contrast advantage of light and the high resolution advantage of ultrasound. In other words, PAT overcomes the resolution disadvantages of pure-optical imaging and the contrast and speckle disadvantages of pure-ultrasonic imaging. Having achieved non-invasive photoacoustic tomography of small-

${ }^{*}$ Corresponding author: email: Lwang@ tamu.edu; phone: 1979 847-9040; fax: 1979 845-4450 
animal brains based on intrinsic optical contrast, ${ }^{8,9}$ we turned our attention to the in vivo functional photoacoustic imaging of cerebral blood oxygenation in small-animal brains, and this work will be presented here.

\section{RECONSTRUCTION ALGORITHM}

We are interested in tissues that have inhomogeneous optical absorption but relatively homogeneous acoustic properties. When the laser pulse is very short, which is the case in our experiments, the time required for thermal diffusion is much greater than the time required for thermoacoustic transition. Consequently, the effect of heat conduction in the thermoacoustic wave equations can be ignored. As has been described in many previous articles, the generation of a photoacoustic wave by the deposition of light energy can be expressed as: ${ }^{10}$

$$
\nabla^{2} p(\mathbf{r}, t)-\frac{1}{c_{s}^{2}} \frac{\partial^{2}}{\partial t^{2}} p(\mathbf{r}, t)=-\frac{\beta}{C_{p}} \frac{\partial S(\mathbf{r}, t)}{\partial t} .
$$

where $p(\mathbf{r}, t)$ is the pressure produced by the photoacoustic source $S(\mathbf{r}, t) ; c_{s}$ is the speed of the acoustic wave; $\beta$ and $C_{p}$ are the isobaric volume expansion coefficient and the heat capacity (per unit mass). Eq. (1) is a typical scalar Helmholtz equation, which can be expressed in the following form based on Green's function,

$$
p(\mathbf{r}, t)=\left.\frac{\beta}{4 \pi C_{p}} \iiint \frac{d^{3} r^{\prime}}{\left|r-r^{\prime}\right|} \frac{\partial S\left(\mathbf{r}^{\prime}, t^{\prime}\right)}{\partial t^{\prime}}\right|_{t^{\prime}=t-\frac{\left|r-r^{\prime}\right|}{c_{s}}} .
$$

The photoacoustic source $S(\mathbf{r}, t)$ can be rewritten in terms of the product of the purely spatial and the purely temporal components:

$$
S(\mathbf{r}, t)=I_{0} A(\mathbf{r}) T(t),
$$

where $A(\mathrm{r})$ describes the light absorption distribution at $\mathbf{r} ; T(t)$ describes the temporal irradiation of the laser; and $I_{0}$ is a factor proportional to the incident radiation intensity. Thus, $p(\mathbf{r}, t)$ can be rewritten as

$$
p(\mathbf{r}, t)=\frac{I_{0} \beta}{4 \pi C_{p}} \iiint \frac{d^{3} r^{\prime}}{\left|r-r^{\prime}\right|} A\left(\mathbf{r}^{\prime}\right) T^{\prime}\left(t^{\prime}\right) .
$$

From this equation, we can see that by keeping the structure of the absorption source unchanged, the acoustic pressure produced by that source is proportional to its absorption coefficient.

Let's consider two-dimensional imaging of optical absorption in a cross-section of the sample through a circular-scan of the photoacoustic signals around this imaged cross-section. When the scanning radius in the circular-scan configuration is much greater than the photoacoustic wavelengths (true in our experiments), the modified back-projection algorithm for $2 \mathrm{D}$ imaging has the form of ${ }^{11}$

$$
A(\mathbf{r})=-\left.\frac{r_{0}^{2} C_{p}}{2 \pi I_{0} c_{s}^{4} \beta} \int_{\theta_{0}} d \theta_{0} \frac{1}{t} \frac{\partial p\left(\mathbf{r}_{0}, t\right)}{\partial t}\right|_{t=\mid \mathbf{r}_{0}-\mathbf{r} / / c_{s}},
$$

which is an integral over the scanning angle $\theta_{0}$. Employing full-view circular-scan geometry, the point-to-point absorption distribution in the rat cerebral cortex can be accurately reconstructed from the detected photoacoustic signals with Eq. (5).

\section{EXPERIMENTAL SETUP}

The experimental setup for functional photoacoustic imaging of cerebral blood oxygenation in the rat brain is shown in Fig. 1. A dye laser (ND6000, Continuum) pumped by an Nd:YAG laser (Brilliant B, Bigsky) provided laser pulses with tunable wavelengths. The FWHM of the laser pulse duration was $6.5 \mathrm{~ns}$; the pulse repetition rate was $10 \mathrm{~Hz}$. The laser beam was expanded and homogenized by a concave lens and a piece of ground glass before it reached the rat head. The incident energy density of the laser beam was controlled to $<3 \mathrm{~mJ} / \mathrm{cm}^{2}$ on the surface of the rat head, which induced a temperature rise in the brain vessels estimated to be $<4 \mathrm{mK}$. An ultrasonic transducer (XMS-310, Panametrics) was driven by a computer-controlled step motor to scan around the rat brain cortical surface in the $x-y$ plane. The photoacoustic signals detected by the ultrasonic transducer were sent to an oscilloscope (TDS 540B, Tektronics) 
through an amplifier. Finally, the digitized photoacoustic signals were collected by the computer for image reconstruction.

Sprague Dawley rats ( 150 g, Charles River Breeding Laboratories) were employed in this work. Before imaging, the hair on each rat's head was removed using hair remover lotion. A dose of $87 \mathrm{mg} / \mathrm{kg}$ Ketamine plus Xylasine $13 \mathrm{mg} / \mathrm{kg}$, administered intramuscularly, was used to anesthetize the rat during the experiment. The rat was fixed by a homemade animal holder with its head protruding into the water tank through a hole in the bottom of the tank. A piece of clear membrane between the water and the rat head sealed the hole. Through a breathing mask, the rat was provided with mixed gas including oxygen $\left(\mathrm{O}_{2}\right)$, carbon dioxide $\left(\mathrm{CO}_{2}\right)$, and nitrogen $\left(\mathrm{N}_{2}\right)$. The concentration of the oxygen in the mixed gas was adjusted through a multi gas flow meter. The applied gas flow to the rat was for $0.61 / \mathrm{min}$. During the in vivo experiment, the sensor of a pulse-oximeter $(8600 \mathrm{~V}$, Nonin Medical, Inc.) clamped a front leg of the rat to monitor the global arterial blood oxygenation.

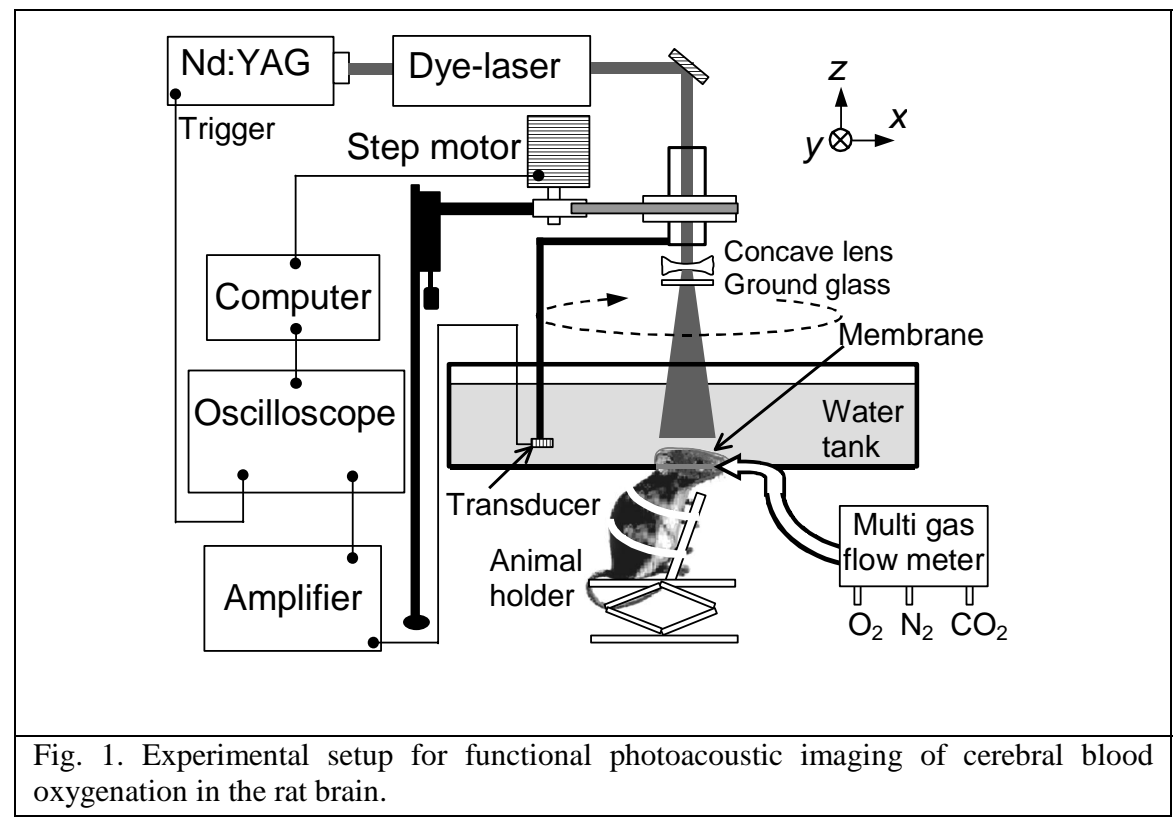

\section{HEMOBLOGIN OXYGEN SATURATION}

Optical measurement of blood oxygen saturation relies on the spectroscopic difference between oxy- and deoxyhemoglobin. For instance, the absorption coefficients of a blood sample at two wavelengths, $\lambda_{1}$ and $\lambda_{1}$, can be expressed as

$$
\left\{\begin{array}{l}
\mu_{a}^{\lambda_{1}}=\varepsilon_{\mathrm{Hb}}^{\lambda_{1}}[\mathrm{Hb}]+\varepsilon_{\mathrm{HbO}_{2}}^{\lambda_{1}}\left[\mathrm{HbO}_{2}\right] \\
\mu_{a}^{\lambda_{2}}=\varepsilon_{\mathrm{Hb}}^{\lambda_{2}}[\mathrm{Hb}]+\varepsilon_{\mathrm{HbO}_{2}}^{\lambda_{2}}\left[\mathrm{HbO}_{2}\right]
\end{array},\right.
$$

where $\mu_{a}\left(\mathrm{~cm}^{-1}\right)$ is the absorption coefficient; $\lambda_{1}$ and $\lambda_{2}$ are the two wavelengths; $\varepsilon_{\mathrm{Hb}}$ and $\varepsilon_{\mathrm{HbO}_{2}}$ are the known molar extinction coefficients $\left(\mathrm{cm}^{-1} \mathrm{M}^{-1}\right)$ of the oxy- and deoxy-hemoglobin, respectively; and $[\mathrm{Hb}]$ and $\left[\mathrm{HbO} \mathrm{Hb}_{2}\right.$ are, respectively, the concentrations $(\mathrm{M})$ of the two forms of hemoglobin. By using the detected absorption coefficients of a blood sample at two wavelengths, we can compute the concentrations of the two forms of hemoglobin by solving the following equation: ${ }^{12-15}$ 


$$
\left\{\begin{array}{c}
{\left[\mathrm{HbO}_{2}\right]=\frac{\varepsilon_{\mathrm{Hb}}^{\lambda_{2}} \mu_{a}^{\lambda_{1}}-\varepsilon_{\mathrm{Hb}}^{\lambda_{1}} \mu_{a}^{\lambda_{2}}}{\varepsilon_{\mathrm{Hb}}^{\lambda_{2}} \varepsilon_{\mathrm{HbO}}^{\lambda_{1}}-\varepsilon_{\mathrm{Hb}}^{\lambda_{1}} \varepsilon_{\mathrm{HbO}_{2}}^{\lambda_{2}}}} \\
{[\mathrm{Hb}]=\frac{\varepsilon_{\mathrm{HbO}_{2}}^{\lambda_{1}} \mu_{a}^{\lambda_{2}}-\varepsilon_{\mathrm{HbO}_{2}}^{\lambda_{2}} \mu_{a}^{\lambda_{1}}}{\varepsilon_{\mathrm{Hb}}^{\lambda_{2}} \varepsilon_{\mathrm{HbO}_{2}}^{\lambda_{1}}-\varepsilon_{\mathrm{Hb}}^{\lambda_{1}} \varepsilon_{\mathrm{HbO}_{2}}^{\lambda_{2}}}}
\end{array}\right.
$$

Then the hemoglobin oxygen saturation is given by

$$
\frac{\left[\mathrm{HbO}_{2}\right]}{\left[\mathrm{HbO}_{2}\right]+[\mathrm{Hb}]}=\frac{\mu_{a}^{\lambda_{2}} \varepsilon_{\mathrm{Hb}}^{\lambda_{1}}-\mu_{a}^{\lambda_{1}} \varepsilon_{\mathrm{Hb}}^{\lambda_{2}}}{\mu_{a}^{\lambda_{1}}} \varepsilon_{\Delta \mathrm{Hb}}^{\lambda_{2}}-\mu_{a}^{\lambda_{2}} \varepsilon_{\Delta \mathrm{Hb}}^{\lambda_{1}} .
$$

By using the photoacoustic images that represent the distributions of the optical absorptions corresponding to the two wavelengths, we can simulate the spatially distributed blood oxygenation with satisfactory resolution and accuracy.

The molar extinction spectra of oxy- and deoxy-hemoglobin are shown in Fig. 2, where a suitable spectrum region for this work is between $500 \mathrm{~nm}$ and $600 \mathrm{~nm}$. In this region, the contrast between brain blood vessels and background brain tissues is high enough to enable satisfactory imaging quality. Meanwhile, the penetration depth of light in this region is greater than the size of most of the blood vessels in the rat brain.

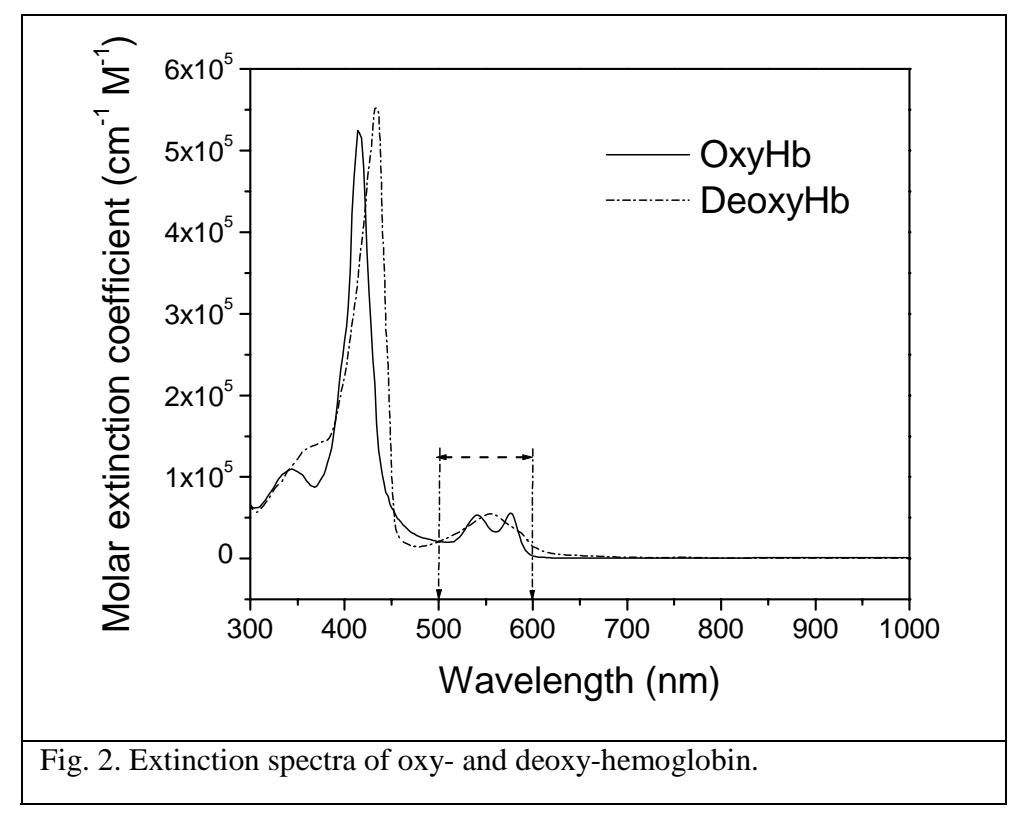

\section{RESULTS}

Functional photoacoustic imaging of cerebral blood oxygenation in the rat brain in vivo was performed with our system. Firstly, pure oxygen $\left(100 \% \mathrm{O}_{2}\right)$ was provided to the rat. In this situation, the rat was under hyperoxia status and its arterial blood oxygenation was measured by the pulse-oximeter to be $\sim 99 \%$. Under this status, two photoacoustic images of the optical absorption in the rat brain cerebral cortex were acquired with the laser light at 581-nm and 600-nm wavelengths, respectively [see Figs 3(A) and (B)]. Then the breathing gas was changed slowly from pure oxygen to a mixed gas with a low concentration of oxygen $\left(\sim 8 \% \mathrm{O}_{2}, \sim 5 \% \mathrm{CO}_{2}\right.$ and $\left.\sim 87 \% \mathrm{~N}_{2}\right)$. At this point, the rat was under hypoxia status. The arterial blood oxygenation level measured by the pulse-oximeter dropped to $\sim 70 \%$. Under hypoxia status, another two photoacoustic images of the rat brain cerebral cortex, corresponding to the two wavelengths $(581 \mathrm{~nm}$ 
and $600 \mathrm{~nm}$ ), were acquired [see Figs 3(C) and (D)]. Hence, totally, we acquired four images corresponding to the two statuses at two wavelengths. With the high optical contrast between the blood and background brain tissues, each brain image presented the vascular structure in the rat cerebral cortex clearly and matched well with the open-skull photograph of the anatomy that we obtained after the imaging experiment [see Fig. 3(H)].

Before the calculation of the blood oxygen saturation, the area of large blood vessels in each image was segmented from the background. The threshold for each image was the averaged optical absorption in the imaged brain area. Then, the point-to-point hemoglobin oxygen saturation levels in the area of the large blood vessels were simulated with Eq. (8). Two functional images of cerebral blood oxygenation corresponding to the hyperoxia and hypoxia statuses are shown in Figs. 3(E) and (F), respectively. These two images use the same gray scale, where darker areas have higher oxygen saturation levels. We can see that when the status of the rat changed from hyperoxia to hypoxia, the blood oxygenation decreased in the rat cerebral cortex.

In order to show this change more clearly, we subtracted the image in Fig. 3(E) from the image in Fig. 3(F). The differential image in Fig. 3(G) presents the change of cerebral blood oxygenation when the status of the rat alternated from hyperoxia to hypoxia. The light areas have negative values, which means that the blood oxygen saturation decreased in the area of the brain vessels as a result of this alternation.

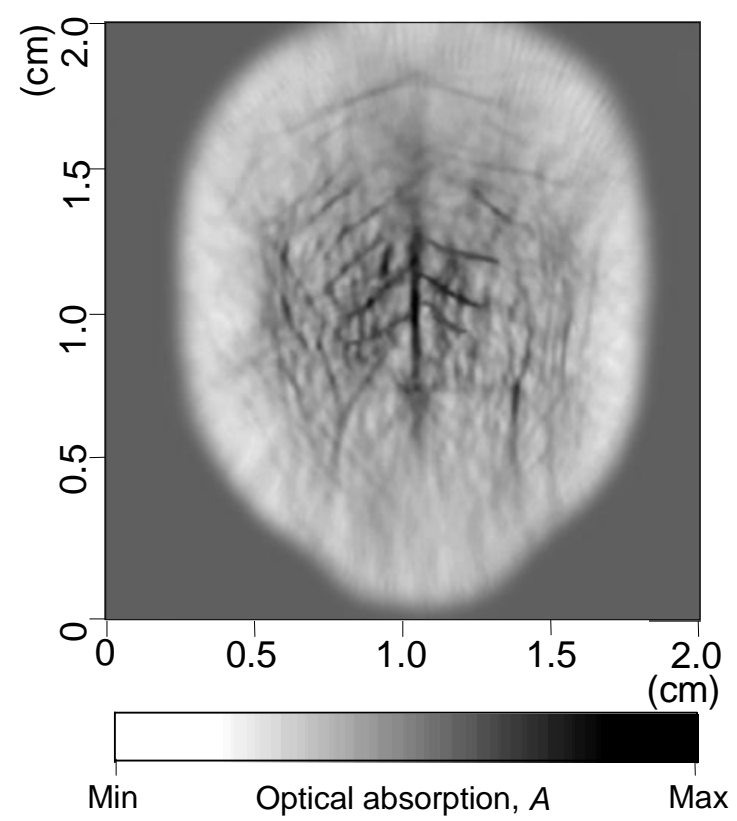

(A)

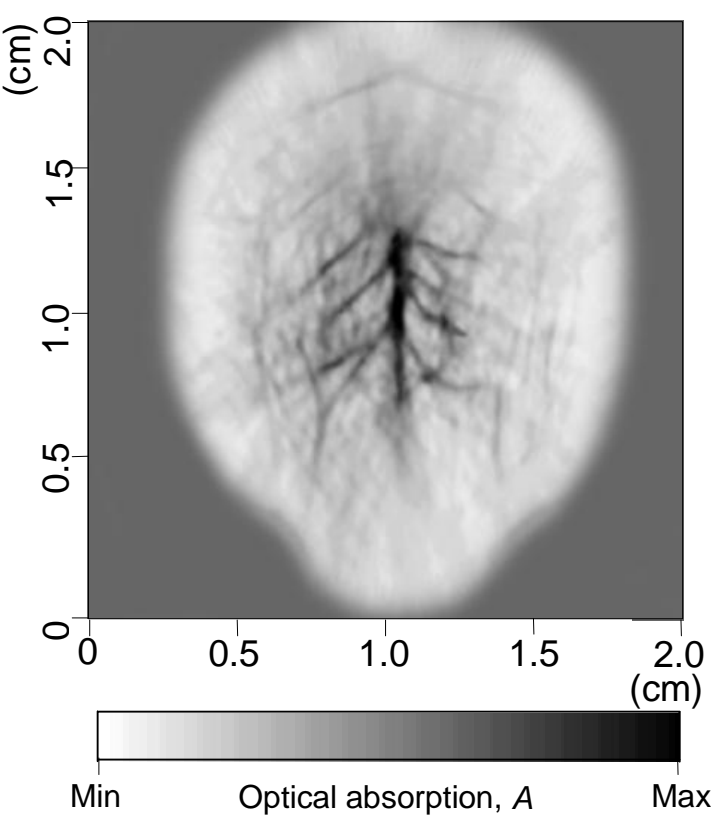

(B) 


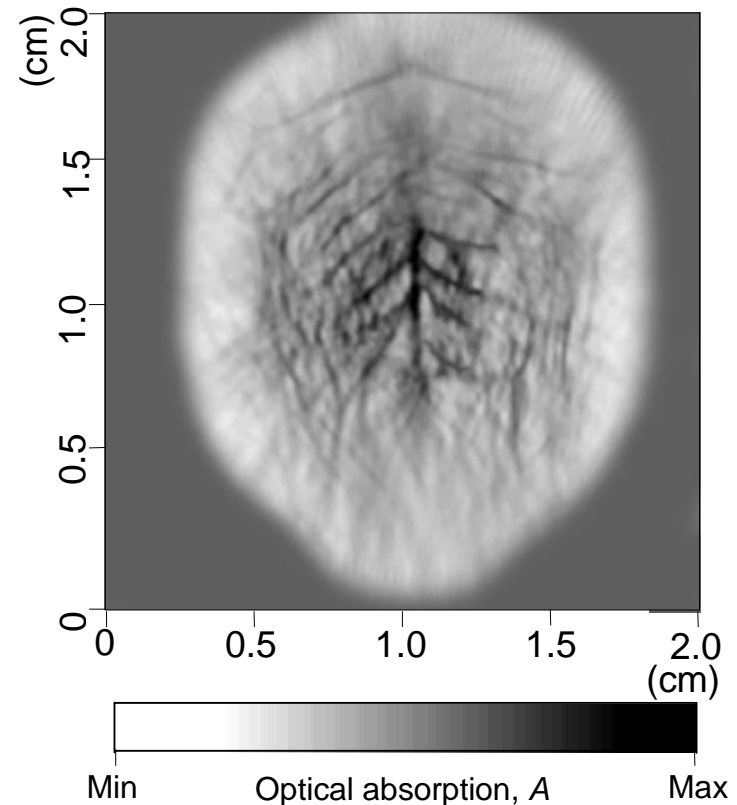

(C)

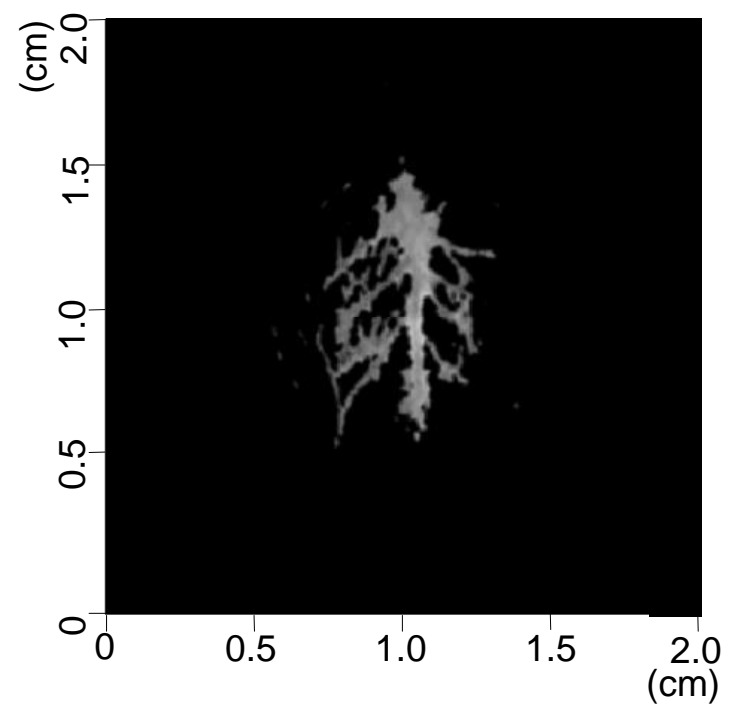

(E)

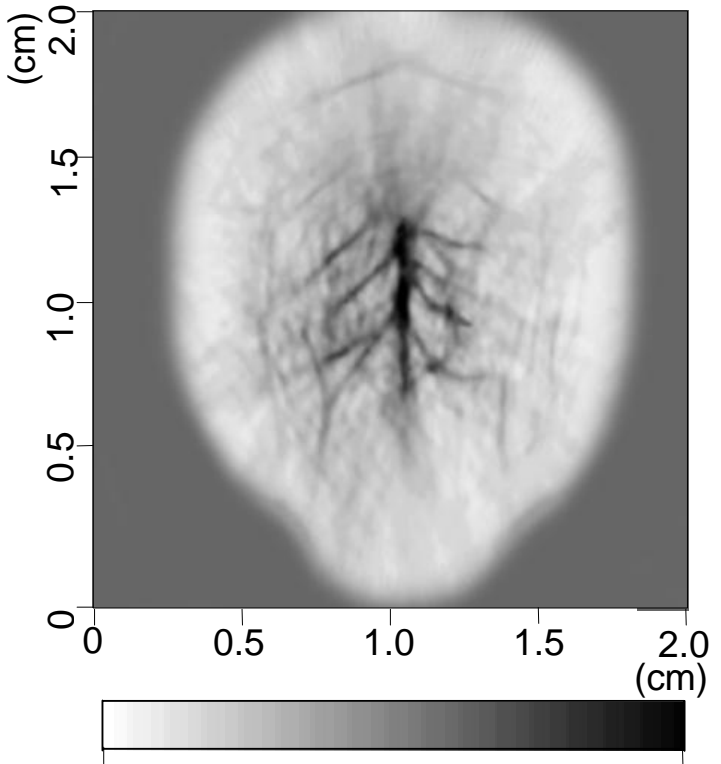

Min $\quad$ Optical absorption, $A \quad$ Max

(D)

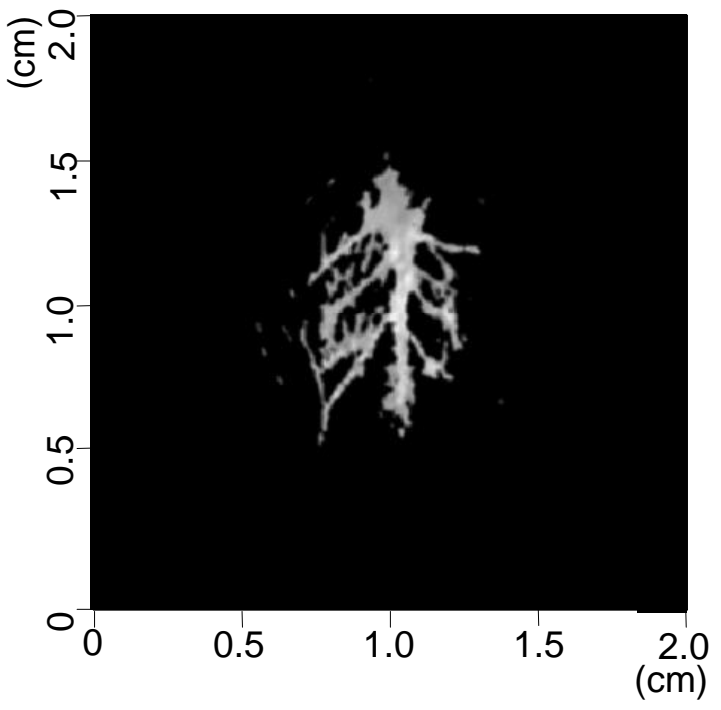

(F)

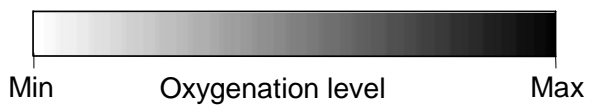




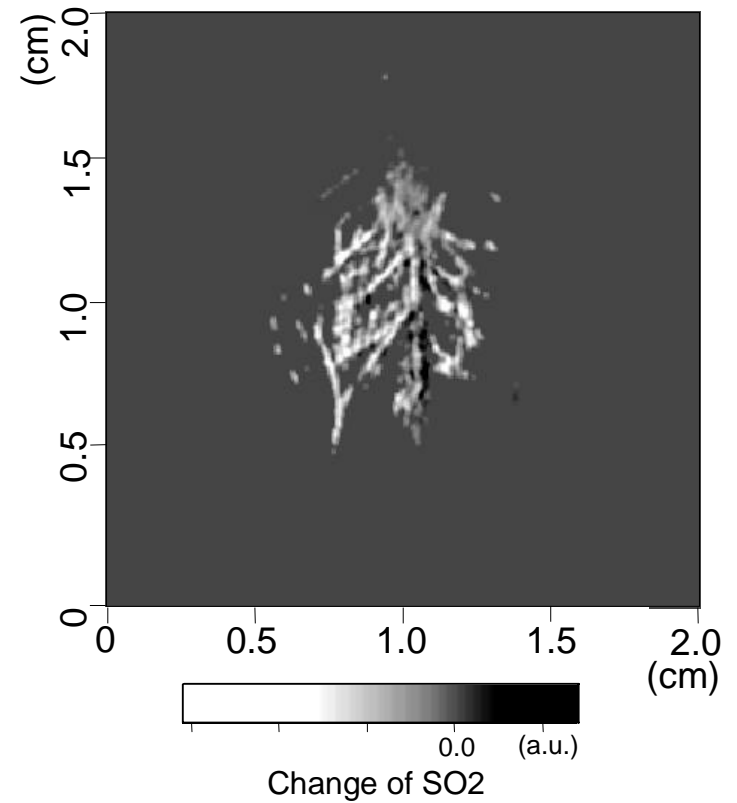

(G)

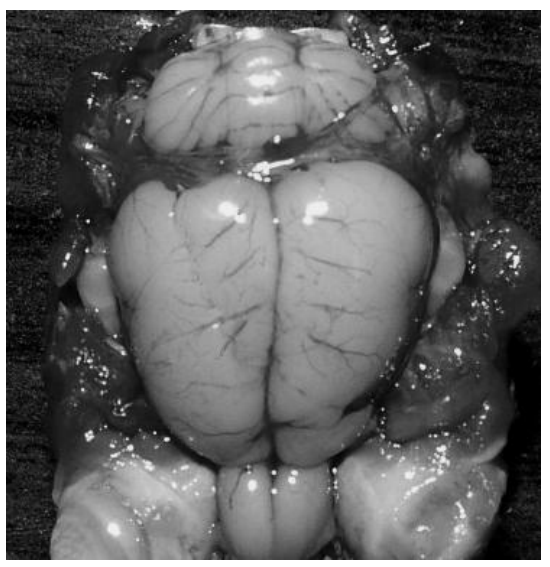

$(\mathrm{H})$

Fig. 3. Functional photoacoustic imaging of cerebral blood oxygenation in a rat brain in vivo. (A) and (B), brain images under the hyperoxia status corresponding to the 581-nm and 600-nm wavelengths, respectively. (C) and (D), brain images under the hypoxia status corresponding to the $581-\mathrm{nm}$ and $600-\mathrm{nm}$ wavelengths, respectively. (E) and (F), Functional images of cerebral blood oxygen saturation in the rat brain under hyperoxia and hypoxia statuses, respectively. (G) Differential image obtained by F minus E, which presents the change of blood oxygen saturation in the rat cerebral cortex when the status of the rat alternated from hyperoxia to hypoxia. $(\mathrm{H})$ Open-skull anatomical photograph of the rat brain obtained after the imaging experiment.

\section{CONCLUSION}

For the first time to our knowledge, photoacoustic tomography was employed to image cerebral blood oxygenation in the small-animal brain in vivo. Since the photoacoustic technique can assess the oxy- and deoxy-hemoglobin simultaneously in a localized area with high sensitivity and specificity, the point-to point hemoglobin oxygen saturation in organs, such as the brain, can be monitored accurately. Non-invasive functional images acquired by our photoacoustic system present a much-improved spatial resolution compared to traditional near-infrared spectroscopy. In addition to blood oxygenation, the photoacoustic technique, based on the spectroscopic differences between the oxyand deoxy-hemoglobin, also shows promise for monitoring changes in the total concentration of hemoglobin (blood flow) in biological tissues. This technique has potential for wide application in neurophysiology, neuropathology and neurotherapy.

\section{REFERENCES}

1. G. A. Millikan, "The oximeter, an instrument for measuring continuously the oxygen saturation of arterial blood in man,” Rev. Sci. Instrum. 13, 434-444 (1942).

2. F. F. Jöbsis, "Noninvasive, infrared monitoring of cerebral and myocardial oxygen sufficiency and circulatory parameters," Science 198, 1264-1267 (1977).

3. A. Villringer and B. Chance, "Non-invasive optical spectroscopy and imaging of human brain function," Trends. Neurosci. 20, 435-442 (1997).

4. C. G. A. Hoelen, F. F. M. de Mul, R. Pongers, and A. Dekker, "Three-dimensional photoacoustic imaging of blood vessels in tissue," Opt. Lett. 23, 648-650 (1998). 
5. R. A. Kruger, D. R. Reinecke, and G. A. Kruger, "Thermoacoustic computed tomography-technical considerations," Med. Phys. 26, 1832-1837 (1999).

6. R. O. Esenaliev, A. A. Karabutov, and A. A. Oraevsky, "Sensitivity of laser opto-acoustic imaging in detection of small deeply embedded tumors," IEEE J. Sel. Top. Quant. 5, 981-988 (1999).

7. K. P. Köstli, D. Frauchiger, J. J. Niederhauser, G. Paltauf, H. P. Weber, and M. Frenz, "Optoacoustic imaging using a three-dimensional reconstruction algorithm," IEEE J. Sel. Top. Quant. 7, 918-923 (2001).

8. X. Wang, Y. Pang, G. Ku, X. Xie, G. Stoica, and L. V. Wang, "Non-invasive laser-induced photoacoustic tomography for structural and functional imaging of the brain in vivo," Nat. Biotech. 21, $803-806$ (2003).

9. X. Wang, Y. Pang, G. Ku, G. Stoica, and L. V. Wang, "Three-dimensional laser-induced photoacoustic tomography of mouse brain with the skin and skull intact," Opt. Lett. 28, 1739-1741 (2003)

10. G. J. Diebold, T. Sun, and M. I. Khan, "Photoacoustic waveforms generated by fluid bodies," in Photoacoustic and Photothermal Phenomena III, edited by D. Bicanic (Springer-Verlag, Berlin, Heidelberg, 1992), pp. 263-269.

11. M. Xu and L. V. Wang, "Time-domain reconstruction for thermoacoustic tomography in a spherical geometry," IEEE T. Med. Imag. 21, $814-822$ (2002).

12. H. Liu, D. A. Boas, Y. Zhang, A. G. Yodh, and B. Chance, "Determination of optical properties and blood oxygenation in tissue using continuous NIR light," Phys. Med. Biol. 40, 1983-1993 (1995).

13. R. L. Barbour, A. Gebrewold, and B. M. Altura, "Optical spectroscopy and cerebral vascular effects of alcohol in the intact brain: effects on tissue deoxyhemoglobin, blood content, and reduced cytochrome oxidase, " Alcohol. Clin. Exp. Res. 17, 1319-1324 (1993).

14. B. Chance, E. Borer, A. Evans, G. Holtom, J. Kent, M. Maris, K. Mccully, J. Northrop, and M. Shinkwin, "Optical and nuclear-magnetic-resonance studies of hypoxia in human tissue and tumors, " Ann. NY Acad. Sci. 551, 1-16 (1988).

15. E. Gratton, S. Fantini, M. A. Franceschini, G. Gratton, and M. Fabiani, "Measurements of scattering and absorption changes in muscle and brain," Philos. T. Roy. Soc. B 352, 727-735 (1997). 